\title{
A Influência de Fatores Sociais, Individuais e Lingüísticos no Desempenho de Crianças na Leitura em Voz Alta de Palavras Isoladas
}

\author{
The Influence of Social, Individual and Linguistic Factors on Children's \\ Performance in Tasks of Reading Single Words Aloud
}

\author{
Patrícia Silva Lúcio* Ângela Maria Vieira Pinheiro \& Elizabeth do Nascimento \\ Universidade Federal de Minas Gerais, Belo Horizonte, Brasil
}

\begin{abstract}
Resumo
O estudo avalia a interferência de fatores sociais, individuais e lingüísticos no desempenho de leitura em voz alta de 333 crianças de Belo Horizonte cursando $1^{\mathrm{a}}$ a $4^{\mathrm{a}}$ série do Ensino Fundamental. Medidas de tempo de reação e precisão foram coletadas. Uma análise de regressão hierárquica (método de Estimação de Equação Generalizada) mostrou a existência dos efeitos de regularidade grafema-fonema e fonemagrafema na leitura e um impacto do número de categorias de regularidade utilizadas na emergência desse efeito. Dos fatores sociais, apenas a escolaridade da mãe foi importante para a precisão (na direção inversa à esperada). Não houve efeito de gênero. Outros fatores além dos tradicionais também mostraramse relevantes, como a idade de aquisição da leitura e a compreensão verbal. O estudo contribuiu com questões teóricas relevantes para a avaliação cognitiva da leitura no português.
\end{abstract}

Palavras-chave: Leitura; Efeito de regularidade; Categorias de regularidade; Fatores sociais.

\begin{abstract}
This study evaluates social, individual and linguistic factors in the performance of a single- word reading aloud task. A group of $1^{\text {st }}$ to $4^{\text {th }}$ grade school children from Belo Horizonte-MG $(N=333)$ read aloud 323 single words presented in a computer screen. Measures of reaction time (RT) and error scores were collected. The Generalized Estimating Equations method exhibited the grapheme-phoneme and phoneme-grapheme regularity effect in reading and also showed an impact on the number of categories of regularity in this effect. No social factor was important to explain the results, but their mothers' education was correlated to the error scores (in opposite direction). There was no gender effect. Other factors rather than the traditional ones were also relevant, such as the age of reading acquisition and the verbal comprehension. The work brings important theoretical issues to cognitive reading assessment in Brazil. Keywords: Reading; Regularity effect; Categories of regularity; Social factors.
\end{abstract}

Após vários anos de estudos da área de reconhecimento de palavras, tem sido documentada uma vasta literatura sobre interferência de fatores diversos no desempenho na leitura. Estes fatores vão desde as características do estímulo apresentado, como a freqüência de ocorrência da palavra ou o número de letras (p. ex., Pinheiro \& Rothe-Neves, 2001), até propriedades individuais e sociais do participante, como a capacidade de memória de trabalho (p. ex., Morais, 1996) e o nível

\footnotetext{
${ }^{*}$ Endereço para correspondência: Rua Costa Rica, Nº. 455 , Bairro Nações Unidas, Sabará, MG, Brasil, CEP 34590-380. Tel.: (31) 3499-6268. E-mail: pslucio@gmail.com Esta pesquisa é parte da dissertação de mestrado da primeira autora, orientada pela segunda e terceira autoras. Agradecemos ao apoio financeiro da Fundação de Amparo à Pesquisa do Estado de Minas Gerais (FAPEMIG) e do Conselho Nacional de Desenvolvimento Científico e Tecnológico (CNPq).
}

socioeconômico (p. ex., Soares \& Collares, 2006). No Brasil, vários estudos envolvendo a tarefa de leitura em voz alta de palavras isoladas têm considerado cada um desses elementos de análise (p. ex., Godoy, 2005; Pinheiro, 2006; Salles, 2005), mas nenhum até o presente considerou a influência simultânea de vários desses fatores. Sendo assim, o objetivo do presente trabalho é investigar a interferência relativa de fatores sociais, individuais e lingüísticos no desempenho na leitura de crianças cursando as séries iniciais do Ensino Fundamental da cidade de Belo Horizonte.

Dos fatores lingüísticos, as influências mais bem documentadas têm sido os efeitos de regularidade, freqüência e extensão (ou de comprimento) na leitura (Coltheart, Rastle, Perry, Langdon, \& Ziegler, 2001; Godoy, 2005; Pinheiro, 2006; Salles, 2005; Salles \& Parente, 2002, 2007; Sucena \& Castro, 2005). A regularidade e o comprimento da palavra afetam a leitura fonológica, a qual 
converte serialmente uma seqüência de grafemas em fonemas, o que leva a um maior tempo de processamento na leitura de palavras grandes ${ }^{1}$ e aumenta as chances de erro nas palavras que apresentam grafemas com pronúncia arbitrária (Coltheart \& Rastle, 1994). Quando a leitura é afetada pela frequiência da palavra, sendo as palavras familiares lidas de forma mais acurada do que as palavras pouco frequientes, há indícios de que as representações lexicais do primeiro grupo de estímulos estejam mais consolidadas do que as do segundo, o qual pode, inclusive, ser lido por mediação fonológica (McCann \& Besner, 1987).

Concentrando nossa atenção especificamente para o efeito de regularidade, Lúcio (2008) e Lúcio e Pinheiro (in press) levantaram algumas questões importantes a respeito da sua emergência no português do Brasil. Para as autoras, os estudos brasileiros apontam para duas questões teóricas importantes: primeiro, o aparecimento do efeito de regularidade na leitura parece ser influenciado pela classificação de regularidade adotada, ou seja, se a direção desta classificação é a da leitura ou a da escrita (Pinheiro \& Rothe-Neves, 2001). Em segundo lugar, há indícios de que o número de categorias utilizadas no estudo também possa interferir nos resultados. Geralmente, o contraste utilizado pelos pesquisadores é o de palavras regulares versus irregulares (p. ex., Godoy, 2005), sendo possíveis outros contrastes, como ao de palavras regidas por regras contextuais (p. ex., Pinheiro, 2006) ou ainda palavras que, além de uma regra contextual, apresentam correspondência irregular, como, aceso (Pinheiro, 2007b).

Em relação às características individuais dos participantes, diversos fatores são apontados como possíveis agentes determinantes do desempenho na leitura. Um desses fatores é a idade de aquisição da leitura, ou seja, a idade em que a criança aprende as primeiras palavras, independentemente do tempo de escolarização. Segundo Zevin e Seidenberg (2002), este fator parece ser relevante para o desempenho na leitura, pois, uma vez algumas palavras são aprendidas antes de outras, a aprendizagem mais anterior torna a representação desses itens mais fortalecida. Para os autores, este efeito se confunde, mas não se sobrepõe, ao efeito de freqüência, uma vez que, controlada a frequiência da palavra, a idade de aquisição ainda permanece importante para a explicação do desempenho na leitura (Zevin \& Seidenberg, 2002).

Vários autores defendem que a memória de trabalho, sobretudo a memória de trabalho verbal (p. ex., Siegel \& Linder, 1984), tem um papel importante na aquisição da leitura. Tem sido bem documentada a relação entre a

${ }^{1} \mathrm{O}$ efeito de extensão ocorre de forma menos pronunciada na leitura lexical, pois esta, diferentemente da leitura fonológica, por ocorrer de forma paralela, recupera a pronúncia e o significado de maneira automática, sendo, portanto, menos afetada pelo número de letras que compõe a palavra. performance em tarefas complexas de memória de trabalho (que envolvem armazenamento de determinada informação enquanto o processamento de informações adicionais se completa) e o desempenho acadêmico entre crianças normais, inclusive no reconhecimento de palavras (p. ex., Leather \& Henry, 1994). Em relação às crianças com dificuldades específicas de aprendizagem (como a dislexia), a discussão é se suas deficiências de memória de trabalho são gerais (p. ex., de Jong, 1998) ou se estas se referem a algum dos componentes específicos da memória requeridos pelas tarefas (p. ex., Bayliss, Jarrold, Baddeley, \& Leigh, 2005). Os estudos com crianças que apresentam dificuldades gerais de aprendizagem são menos numerosos, mas Bayliss et al. (2005) mostraram que a memória de trabalho não se relaciona com o reconhecimento de palavras ou com a compreensão na leitura deste grupo de crianças.

Outro fator individual constantemente relacionado à habilidade de leitura é a consciência fonológica, definida de uma maneira ampla como a habilidade de perceber e manipular, de maneira explícita e ativa, os sons que compõem as palavras faladas (Morais, 1996). A consciência fonológica tem sido intensivamente investigada desde o estudo pioneiro de Liberman (1973, citado por Castles \& Coltheart, 2004). Em uma revisão de literatura recente, Castles e Coltheart (2004) expõem que grande parte do interesse no tema deve-se à hipótese inicial de que a consciência fonológica teria um papel causal na aquisição da leitura. Entretanto, a partir da análise dos estudos longitudinais publicados na língua inglesa até então, os autores afirmam não terem encontrado evidências de que a consciência fonológica precede e influencia diretamente o desenvolvimento da leitura. Segundo os autores, a ausência de evidências desse tipo se deve a problemas metodológicos das pesquisas, as quais, na maioria das vezes, não controlaram a pré-existência de habilidades de leitura entre os participantes, confundindo, assim, os conceitos de consciência fonológica e de habilidades ortográficas. Além disso, nenhum dos estudos de treinamento de habilidades fonológicas em crianças completamente pré-letradas demostrou de forma efetiva que aquelas submetidas ao treinamento apresentaram habilidades de leitura e de escrita melhores do que as que não foram submetidas. Sendo assim, apesar de ser inquestionável a existência de um relacionamento entre esta habilidade e a aquisição da leitura, a natureza dessa relação e sua direção ainda não foram claramente estabelecidas.

Um problema que concerne ao estudo da relação entre a consciência fonológica e a leitura é a interferência da habilidade ortográfica no desempenho das tarefas de consciência fonológica, o que contradiz o princípio de que estas tarefas medem um domínio que se refere especificamente à fala. Isto é consistente com estudos que sugerem que a aprendizagem da leitura desperta o indivíduo para os elementos constituintes da fala (Castles \& 
Coltheart, 2004). Isso implica que a avaliação da habilidade fonológica da criança pode ser confundida com a avaliação de um construto de natureza ortográfica, o que pode se agravar à medida que aumenta o nível de escolarização da criança.

Mais controversa é a participação da inteligência no desempenho na leitura. Durante muitas décadas, o Quociente de Inteligência (QI) foi utilizado como critério diagnóstico para a dislexia e tal postura foi duramente criticada nos últimos tempos (Fuchs \& Yung, 2006). Num primeiro momento, um baixo QI era utilizado como critério de exclusão de problemas específicos de aprendizagem, o que afastava da definição aquelas crianças que, além de uma deficiência intelectual, paralelamente apresentavam problemas específicos de aprendizagem (Gerber, 1996). Posteriormente, um procedimento conhecido como "método da discrepância" passou a ser utilizado, a partir do qual as crianças com um desempenho na leitura aquém do que seria esperado pelo seu potencial (medido pelo nível de inteligência) seriam diagnosticadas como disléxicas. O método da discrepância também tem sido criticado e apresenta como principal problema o pressuposto implícito de que os disléxicos são qualitativamente distintos dos leitores com dificuldades gerais de aprendizagem, o que ainda é controverso entre os pesquisadores (Badian, 1994; Toth \& Siegel, 1994).

Acreditamos que o debate sobre inclusão da avaliação da inteligência em critérios diagnósticos para a dislexia não pode obscurecer a pesquisa sobre as possíveis contribuições da inteligência para o desenvolvimento da leitura. Sabemos que a inteligência apresenta múltiplos domínios (Neisser et al., 1996), sendo que algum deles pode estar relacionado ao desempenho na leitura. De fato, trabalhos têm relacionado medidas de inteligência geral à habilidade de leitura tanto em crianças (p. ex., Stanovich, Cunningham, \& Freeman, 1984) quanto entre adultos (p. ex., Johnson, Bouchard, Segal, \& Samuels, 2005). O uso de testes de inteligência que avaliam diretamente habilidades verbais e outras habilidades cristalizadas, como as Escalas Wechsler de Inteligência para crianças (WISC) e para adultos (WAIS), pode também favorecer o aparecimento de relações com a leitura (Johnson et al., 2005). No entanto, no Brasil, Maia e Fonseca (2002) mostraram que o QI, avaliado pelo WISC, foi ineficiente na predição das habilidades de leitura de palavras simples por crianças de 7 a 15 anos. De maneira semelhante, Dal Vesco, Mattos, Benincá e Tarasconi (1998) não encontraram relação entre o desempenho acadêmico e os escores no WISC em crianças de escolas públicas e particulares.

Diversos fatores socioeconômicos estão associados ao desempenho acadêmico. Em um estudo transcultural conduzido em 43 países, inclusive o Brasil, Chiu e McBride-Chang (2006) mostraram que variáveis socioeconômicas familiares estão positivamente relacionadas com o desempenho na leitura de adolescentes. Juntos, esses fatores explicaram $30 \%$ da variância do desempenho na leitura. Os autores concluíram que todo modelo compreensivo para a aquisição da leitura deve considerar as variáveis peculiares ao país, à família, à escola e às características individuais (como a motivação para a leitura). De maneira análoga, Steensel (2006) mostrou que uma medida do envolvimento do ambiente familiar no letramento causou um efeito no vocabulário da criança e na compreensão da leitura. Enfim, Duncan e Seymour (2000) mostraram que crianças escocesas de baixo nível socioeconômico tiveram um atraso na aquisição da habilidade de leitura em relação aos seus pares de alto nível socioeconômico.

No Brasil, pesquisas têm relacionado variáveis socioeconômicas à produção do fracasso escolar. Carvalho (2004) mostrou que meninos pobres e negros apresentam mais problemas de aprendizagem (representada pela necessidade de reforço escolar) do que seus pares (de ambos os gêneros na cor branca e meninas negras). Em vários países têm sido relatadas diferenças de gênero na leitura, com desempenho favorável para as meninas (p. ex., Chiu \& McBride-Chang, 2006), mas o diferencial do estudo de Carvalho (2004) é a relação deste fator com a etnia da criança. No Brasil, o efeito do tipo de escola no desempenho da criança também tem sido um elemento constantemente relatado por dados oficiais (Soares, 2004).

Tendo em vista a relevância dos fatores aqui reportados para o desempenho na leitura, propõe-se verificar a influência de algumas destas medidas na precisão e no tempo de processamento da leitura em voz alta de palavras isoladas. Das habilidades reportadas, apenas a consciência fonológica não foi avaliada, devido às questões levantadas por Godoy (2001) sobre os instrumentos de avaliação dessa habilidade no Brasil (que demonstrou a inadequação de muitas tarefas de consciência fonológica) e por esta ser fortemente influenciada pelo desenvolvimento ortográfico à medida que a escolarização avança, sendo, portanto, mais útil nos estudos com crianças préescolares (Castles \& Coltheart, 2004). A avaliação da memória de trabalho será representada pelo escore no índice de resistência à distração do WISC-III.

Em relação às características lingüísticas, trataremos dos efeitos de regularidade e extensão, mas concentraremos nossa atenção para o primeiro efeito. Nossa hipótese é de que a regularidade seja o fator lingüístico que mais contribua para as diferenças de desempenho das crianças, mas a direção da classificação de regularidade da palavra deve afetar de forma distinta a precisão e o tempo de reação, conforme sugeriram Lúcio (2008) e Lúcio e Pinheiro (in press). Para as autoras, a precisão é afetada pela regularidade da palavra para a leitura, enquanto que o tempo de reação sofre o efeito da regularidade para a escrita. 


\section{Método}

Este trabalho é parte de um projeto maior, intitulado "Avaliação de Competências Psicológicas da População Infanto-Juvenil de Belo Horizonte: Inteligência e Habilidades de Leitura e Escrita" (Pinheiro, 2007a), que foi aprovado pelo Comitê de Ética em Pesquisa com Seres Humanos (COEP) da Universidade Federal de Minas Gerais ([UFMG], processo n. ${ }^{\circ}$ ETIC 347/04).

\section{Participantes}

Trezentas e trinta e três (333) crianças da $1^{\mathrm{a}}$ a $4^{\mathrm{a}}$ série $^{2}$ do Ensino Fundamental (idade mínima $=6$ anos; idade máxima $=12$ anos; média de idade $=8,77, D P=2,14$; $52 \%$ do sexo feminino), oriundas de sete escolas da rede de ensino de Belo Horizonte (quatro estaduais, duas municipais e uma particular), com nível socioeconômico variando de $\mathrm{A} 2$ a D (Associação Brasileira de Empresas de Pesquisa [ABEP], 2003). A coleta de dados ocorreu de agosto de 2007 a março de 2008.

\section{Instrumentos}

As crianças foram avaliadas quanto à leitura, à inteligência e ao desempenho escolar. Em relação à leitura, foi aplicado um banco de 323 de palavras de baixa freqüência de ocorrência (Pinheiro, 2007b), variando em níveis de regularidade para a leitura e para a escrita (ver próxima seção) e número de letras, aplicado por meio de computadores portáteis. Para fins de aplicação, este foi dividido em duas partes, a Lista 1 e a Lista 2. Em cada aplicação, havia duas pausas pré-programadas, para o caso de a criança necessitar de descanso. As respostas e o tempo de reação das crianças (medido em milésimos de segundo) foram automaticamente gravados pelo computador por meio do programa Cogwork (especialmente cedido pelo pesquisador Philip Seymour da universidade de Dundee, Escócia). Para a avaliação da inteligência, as crianças responderam às Matrizes Progressivas de Raven (Raven, Raven, \& Court, 1993), à escala verbal do WISC-III (Wechsler, 1991). Para a avaliação do desempenho escolar, aplicou-se o Teste de Desempenho Escolar (Stein, 1994; este último teste não será tratado aqui).

Os pais, junto ao termo de consentimento, responderam a um questionário sobre o nível socioeconômico da família (ABEP, 2003) e também uma avaliação subjetiva sobre as dificuldades de aprendizagem da língua portuguesa da criança e a idade aproximada em que aprendeu a ler (que chamaremos aqui de idade de aquisição da leitura). Após as sessões de avaliação, as crianças também responderam a uma auto-avaliação sobre as suas próprias dificuldades de aprendizagem (Lúcio, 2008).
Classificação das Palavras. O estudo adotou, com algumas modificações, a classificação de regularidade bidirecional de palavras introduzida por Pinheiro (2007b). Esta classificação considera a pronúncia de cada grafema e de cada fonema presente nas palavras e estabelece, dentre outras distinções, a que ocorre entre as pronúncias que são dependentes de contexto (p. ex., a pronúncia do $<$ s $>$ na palavra mesa) daquelas que são independentes de contexto (p. ex., cada). No primeiro caso, as palavras são classificadas como "regra" (RG) e, no segundo, como regulares (R). Além disso, a classificação distingue as palavras que apresentam uma combinação de uma (ou mais) correspondência irregular com uma (ou mais) correspondência regida por regras (p. ex., a palavra aceso apresenta uma irregularidade (o grafema $<\mathrm{e}>$ ) e uma regra de pronúncia (o $<$ s $>$ intervocálico), sendo classificada, portanto, como irregular-regra (IRRG) para a leitura). Por fim, as palavras irregulares (IR) são aquelas que apresentam pronúncias ou grafias arbitrárias. É o caso, por exemplo, do $<x>$ intervocálico, que pode apresentar várias pronúncias e pode ser graficamente representado de várias maneiras (irregularidade tipo 1A) e dos grafemas $<\mathrm{e}>\mathrm{e}<\mathrm{o}>\mathrm{em}$ sílaba tônica, que podem ser lidos de forma aberta ou fechada (irregularidade tipo 1B). Na escrita, o número de casos de irregularidade é bem maior do que para a leitura (Parente, Silveira, \& Lecours, 1997).

Como a classificação proposta é bidirecional, as mesmas possibilidades de classificação para a leitura também são feitas para a escrita (no exemplo anterior, a palavra aceso seria classificada como IRRG para a escrita, pois o som /z/ pode ser arbitrariamente escrito com $<\mathrm{z}>$ ou com $<\mathrm{s}>$ e a grafia do som /U/ no final de palavra, escrita com $<0>$, é regida por uma regra contextual). Cabe ressaltar que a regularidade da palavra para a leitura é independente de sua classificação para a escrita e vice-versa, isto é, uma palavra regular para a leitura pode ser regular, regra, irregular ou irregularregra para a escrita, e assim por diante. A principal diferença da classificação adotada no presente estudo em relação à proposta original de Pinheiro (2007b) diz respeito à classificação das vogais, que no presente estudo está de acordo com a apresentada por Silveira $(1986)^{3}$, enquanto que a de Pinheiro está de acordo com a de brasileiro, existe uma discordância entre os lingüistas, sendo que um grupo considera a existência de 26 fonemas (grupo de Matoso Câmara, 1985, citado por Parente et al., 1997) enquanto outro considera a existência de 33 fonemas (grupo de Silveira, 1986). Segundo Parente et al. (1997), a abordagem de Silveira (1986) não modifica as irregularidades nas conversões, sendo que apenas aumenta o número de regras contextuais. No entanto, conforme dito acima, no presente trabalho, a classificação de palavras diferencia entre as pronúncias dependentes de contexto das independentes de contexto, o que nos fez optar pela classificação de Silveira (1986) 
Matoso Câmara (1985, citados por Parente et al., 1997). As demais modificações se referem a casos particulares, e não a um contexto geral, como a opção pela classificação de Silveira (1986) aqui apontada (para maiores detalhes, ver Lúcio, 2008).

\section{Procedimentos de Análise Estatística}

Para a análise estatística, foi utilizado o modelo marginal produzido pelo método de Estimação de Equação Generalizada (GEE) para determinar o peso relativo de cada co-variável selecionada na proficiência na leitura (entende-se por proficiência na leitura, índices satisfatórios ou médios de precisão e tempos de reação). Modelos diferenciados foram reportados para a precisão (modelo GEE logístico) e para o tempo de reação (modelo GEE linear), ambos com uma estrutura homogênea. O método GEE fornece modelos semelhantes ao da regressão hierárquica, com a diferença que não apresenta suposições com relação à distribuição dos efeitos (ou seja, é adequado a dados não paramétricos, como é o caso do presente estudo; para maiores detalhes sobre os modelos GEE, ver Hu, Hedeker, Flay, \& Pentz, 1998). Para a seleção de variáveis dos modelos linear e logístico, foi utilizado o procedimento de stepwise backward. Este método ajusta um modelo completo e, em seguida, retira a variável não significante de menor peso no modelo. Deste modo, o procedimento é repetido até que sobrem apenas variáveis estatisticamente significativas (no presente estudo, aquelas que apresentaram $p<0,05$ ).

\section{Resultados}

\section{Modelo de Tempo de Reação}

A Tabela 1 resume os dados do estudo. Nela são apresentadas as médias e os desvios padrão para o tempo de reação em milisegundos (ms) e para a precisão (\% acertos) das crianças em função da série e no total.

Tabela 1

Valores de Média (M) e Desvio Padrão (DP) para a Proporção de Acertos (\%) e para o Tempo de Reação (ms) na Leitura da Amostra para Cada Série Separadamente e no Total

\begin{tabular}{lrccc}
\hline Série & \multicolumn{2}{c}{ TR } & \multicolumn{2}{c}{ Precisão } \\
\cline { 2 - 5 } & Média & $D P$ & Média & $D P$ \\
\hline $1^{\mathrm{a}}$ & 1399 & 967 & 72 & 45 \\
$2^{\mathrm{a}}$ & 1156 & 781 & 79 & 45 \\
$3^{\mathrm{a}}$ & 957 & 458 & 86 & 35 \\
$4^{\mathrm{a}}$ & 885 & 414 & 87 & 33 \\
Total & 1079 & 710 & 81 & 40 \\
\hline
\end{tabular}

A Tabela 2 apresenta a equação de estimação do tempo de reação e para a precisão, com todas as co-variáveis que se mostraram significativas. Confirmando as expectativas levantadas, foram consideradas preditoras para o tempo de reação (TR) as covariáveis idade de aquisição da leitura, série, número de letras da palavra e a classificação de regularidade da palavra para a escrita. Todas estas covariáveis entraram no modelo de regressão na direção esperada.

No que diz respeito às características do participante, observou-se que cada ano de atraso na aquisição da leitura corresponde a um aumento de cerca de $114 \mathrm{~ms}$ no TR da criança. Isso indica que, quanto mais cedo a criança aprende a ler as primeiras palavras, maior a sua rapidez em termos de tempo de processamento. Além disso, foi observado um forte efeito de escolaridade: passando da primeira para a segunda série, há uma redução de quase $373 \mathrm{~ms}$ no TR. Uma redução de $474 \mathrm{~ms}$ no TR é observada da primeira para a terceira série, enquanto que nas comparações com a quarta série a redução chegou aos $685 \mathrm{~ms}$. Todas as comparações da primeira série com as demais foram significativas.

Contrariando as expectativas, o fator de resistência à distração não foi relevante para explicar os resultados. Dos fatores de inteligência, apenas a compreensão verbal mostrou-se relevante para o modelo, havendo uma redução de $8 \mathrm{~ms}$ para cada unidade de aumento no índice de compreensão verbal (ICV). Nenhuma das variáveis sociais contribuiu para a equação, inclusive o nível socioeconômico da criança. Não houve efeito de gênero.

Para as características da palavra, houve um forte efeito de extensão, sendo que para cada unidade de aumento da palavra (em número de letras) houve um acréscimo de $63 \mathrm{~ms}$ no TR. Corroborando nossa hipótese, apenas a classificação de regularidade para a escrita da palavra foi relevante para as variações no TR. Além disso, o modelo com quatro categorias de regularidade foi o que mais bem se adequou aos dados, tornando evidente a influência da quantidade de categorias adotadas no trabalho na produção de efeitos na leitura. As palavras regulares foram lidas com mais rapidez do que as palavras irregulares (IR e IRRG), mas as comparações com as palavras da categoria regra não foram significativas. O aumento do TR das demais classificações em comparação com as palavras regulares variou de seis a $52 \mathrm{~ms}$. Ao contrário do que foi esperado, houve uma forte influência da ordem na direção de um efeito de fadiga no TR. A cada palavra lida, houve um aumento médio de $0,50 \mathrm{~ms}$ no TR.

\section{Modelo de Precisão}

Para o modelo de precisão, mais variáveis foram consideradas preditoras do que o modelo de TR, mas de maneira análoga a este último, não houve efeito de gênero na precisão da leitura. Em comum, a idade de aquisição da leitura, a série, o ICV, o número de letras da palavra e a regularidade para a escrita da palavra. Adi- 
Lúcio, P. S., Pinheiro, A. M. V. \& Nascimento, E. (2010). A Influência de Fatores Sociais, Individuais e Lingüísticos no Desempenho de Crianças na Leitura em Voz Alta de Palavras Isoladas.

Tabela 2

Equação de Estimação para o Tempo de Reação e para a Precisão na Leitura

\begin{tabular}{|c|c|c|c|}
\hline Indicador & Coeficiente & $D P$ & $P$-valor \\
\hline \multicolumn{4}{|l|}{ Modelo de TR } \\
\hline Ordem de apresentação & 0,50 & 0,10 & $<0,0001$ \\
\hline Idade de aprendizagem da leitura (anos) & 114,02 & 48,36 & $<0,02$ \\
\hline Série $\left(1^{\mathrm{a}}\right.$ série $)$ & & & $<0,0001$ (conj.) \\
\hline $2^{\mathrm{a}}$ série & $-372,47$ & 149,70 & $<0,03$ \\
\hline $3^{a}$ série & $-474,32$ & 126,68 & $<0,0001$ \\
\hline $4^{\mathrm{a}}$ série & $-685,09$ & 140,64 & $<0,0001$ \\
\hline ICV (escore) & $-7,80$ & 2,24 & $<0,0001$ \\
\hline Número de letras & 62,64 & 5,97 & $<0,0001$ \\
\hline Classificação para a escrita (regular) & & & $<0,0001$ (conj.) \\
\hline Regra & 6,27 & 3,96 & 0,11 (n.s.) \\
\hline Irregular & 43,75 & 5,89 & $<0,0001$ \\
\hline Irregular-regra & 52,49 & 6,97 & $<0,0001$ \\
\hline \multicolumn{4}{|l|}{ Modelo de precisão } \\
\hline Pré-escola (não ter feito) & 1,77 & 0,39 & $<0,002$ \\
\hline Idade de aprendizagem da leitura (anos) & 0,76 & 0,05 & $<0,0001$ \\
\hline Dificuldade com português (não ter dificuldade) & 0,76 & 0,10 & $<0,04$ \\
\hline Série $\left(1^{\mathrm{a}}\right.$ série $)$ & & & $<0,0001$ (conj.) \\
\hline $2^{\mathrm{a}}$ série & 1,53 & 0,26 & $<0,001$ \\
\hline $3^{\mathrm{a}}$ série & 2,08 & 0,37 & $<0,001$ \\
\hline $4^{\mathrm{a}}$ série & 3,06 & 0,70 & $<0,001$ \\
\hline \multicolumn{4}{|l|}{ Escolaridade da mãe } \\
\hline (analfabeto/primário incompleto) & & & $<0,03$ (conj.) \\
\hline Primário completo/ginasial incompleto & 1,02 & 0,30 & 0,94 (n.s.) \\
\hline Ginasial completo/ colegial incompleto & 0,85 & 0,22 & 0,52 (n.s.) \\
\hline Colegial completo/ superior incompleto & 0,99 & 0,24 & 0,97 (n.s.) \\
\hline Superior completo & 0,50 & 0,14 & $<0,02$ \\
\hline $\mathrm{ICV}$ (escore) & 1,02 & 0,00 & $<0,0001$ \\
\hline Número de letras & 0,91 & 0,01 & $<0,0001$ \\
\hline Classificação para a leitura (regular) & & & $<0,0001$ (conj.) \\
\hline Regra & 0,86 & 0,03 & $<0,0001$ \\
\hline Irregular & 0,58 & 0,02 & $<0,0001$ \\
\hline Irregular-regra & 0,51 & 0,02 & $<0,0001$ \\
\hline Classificação para a escrita (regular) & & & $<0,0001$ (conj.) \\
\hline Regra & 0,80 & 0,02 & $<0,0001$ \\
\hline Irregular & 0,78 & 0,03 & $<0,0001$ \\
\hline Irregular-regra & 0,64 & 0,02 & $<0,0001$ \\
\hline
\end{tabular}

Nota. $D P=$ desvio padrão. $\mathrm{ns}=$ valores não significativos. Entre parêntesis: variáveis indicadoras.

cionalmente, mostraram-se adequadas para o modelo o fato de a criança ter feito pré-escola ${ }^{4}$, a escolaridade da mãe, a auto-avaliação da criança sobre suas próprias dificuldades com a língua portuguesa e a classificação da palavra para a leitura. A ordem não foi relevante para a explicação dos resultados em termos de precisão. A Ta-

${ }^{4}$ Que é uma medida independente da idade de aquisição da leitura, uma vez que a criança pode ter feito pré-escola, mas só ter aprendido as primeiras palavras mais tarde, quando já cursando o ensino fundamental. bela 2 apresenta a equação de estimação da precisão, com todas as co-variáveis que se mostraram significativas.

O modelo GEE logístico apresenta a estimativa do efeito em termos de razão de chance. Primeiramente, serão descritas as influências das características do participante na precisão. A cada ano de atraso na aprendizagem da leitura, as chances de acerto são $24 \%$ menores. Ter feito pré-escola aumenta em $77 \%$ as chances de acerto. O reconhecimento pela criança de suas próprias dificuldades com a aprendizagem do português reduz em $24 \%$ suas chances de acerto. Em relação à escolaridade, passando 
da primeira para a segunda série, as chances de acerto aumentam em $53 \%$. Da primeira para a terceira série, o aumento é de $100 \%$, enquanto que em relação à quarta este aumento da chance de acerto é de $300 \%$. Por fim, a cada unidade de aumento do ICV há um aumento de $2 \%$ na chance de acerto.

Das características sociais, conforme dito acima, apenas a escolaridade materna mostrou-se importante e a direção de sua influência ocorreu de uma maneira inusitada. Com exceção das relações entre os dois níveis mais inferiores de escolaridade, a relação entre escolaridade da mãe e proficiência na tarefa de leitura é inversa, ou seja, quanto maior a escolaridade da mãe, pior o desempenho da criança. A diferença do conjunto foi significativa ao nível de $p<0,03$. Além disso, a única diferença significativa com as comparações com a variável indicadora foi com o nível mais superior de escolaridade da mãe, sendo que a chance de acerto neste nível foi $50 \%$ menor.

A cada aumento no número de letras da palavra, há uma redução de $9 \%$ na chance de acerto (efeito de extensão). Em relação à classificação da palavra para a leitura e para a escrita, passando da categoria regular para as demais categorias, há uma redução significativa da chance de acerto. Diferentemente do modelo de TR, as comparações entre as palavras regulares e as regras foram significativas. No geral, a maior redução da razão de chance ocorreu nas comparações com as palavras IRRG, tanto para a regularidade na leitura quanto para a escrita, sendo a chance de acerto nesses itens respectivamente $49 \%$ e $36 \%$ menor em relação à variável indicadora. A título do que ocorreu com o TR, o modelo com quatro categorias de regularidade foi o que melhor explicou os resultados da amostra.

\section{Discussão}

O presente estudo considerou várias questões relevantes sobre a tarefa de leitura em voz alta no português. No que diz respeito ao efeito de regularidade, uma hipótese lançada por Lúcio (2008) e Lúcio e Pinheiro (in press) é de que a emergência desse efeito na precisão e no tempo de processamento na leitura poderia variar em função do número e do tipo de categorias que se utiliza na pesquisa. De fato, os resultados obtidos aqui mostraram que o uso de quatro categorias de regularidade é mais eficaz para explicar o desempenho das crianças do que o uso de duas categorias apenas. As comparações entre as diversas categorias mostraram que o efeito maior ocorre nas comparações entre as palavras R e as IRRG. Isto sugere que faz sentido a distinção introduzida por Pinheiro (2007b) entre palavras que apresentam apenas correspondências irregulares daquelas que, adicionalmente a uma correspondência irregular, apresentam também correspondências dependentes contexto. Nesse sentido, parece que o primeiro grupo de itens impõe maior demanda de processamento do que o segundo.
Esses resultados, aliados à presença de diferenças significativas na leitura das palavras regulares em relação às palavras regras na precisão, sugerem que o domínio de regras contextuais na leitura pode não se estabelecer tão cedo durante a aprendizagem da língua, como sugerem alguns autores (p. ex., Pinheiro \& Rothe-Neves, 2001). Pelo menos no que se refere aos erros, essas palavras continuam impondo alguma dificuldade aos leitores, dificuldade esta que supera à que é imposta pelos itens cuja pronúncia é independente de contexto. Entretanto, um fato importante a ser mencionado é que a classificação das palavras adotada nesse estudo gerou um maior número de palavras dependentes de regras contextuais do que outros estudos que não encontraram diferenças significativas entre as palavras regulares dependentes e independentes de contexto (p. ex., nos estudos de Pinheiro, 2006, e de Pinheiro, Lúcio, \& Silva, 2008). Este fato reforça a hipótese de que, dependendo da classificação utilizada pelo estudo, os efeitos entre as categorias de palavras podem ser observados ou suprimidos. Além disso, uma análise detalhada dos tipos de erros cometidos na leitura das palavras regra do presente estudo poderá confirmar se o efeito aqui obtido se deve ou não a desconsiderações de regras contextuais.

A hipótese de que o nível de regularidade para a escrita das palavras é relevante para o tempo de reação na leitura recebeu suporte empírico. Ainda não está claro porque esse efeito ocorre, mas é possível que o fato de a aquisição das regras na escrita se efetuar de forma mais lenta e laboriosa do que as regras para a leitura tenha alguma interferência (Parente et al., 1997). Por exemplo, Pinheiro e Rothe-Neves (2001), levantam a hipótese de que as palavras irregulares para a escrita, mas regulares para a leitura, podem causar dificuldades na leitura das crianças mais novas devido ao seu domínio mais fraco de conhecimento das relações fonema-grafema. Para Pinheiro (2006), as palavras contendo, por exemplo, $c, s c, c$ e $x$ para representar $/ \mathrm{s} /$ - especialmente as menos freqüentes - podem ser reconhecidas menos rapidamente do que as soletradas com $s$ (a correspondência regular do fonema /s/) como resultado de pouca experiência com tal correspondência (devemos lembrar que os grafemas $<c ̧>\mathrm{e}<\mathrm{sc}>$ são regulares para a leitura). Nesse sentido, pode-se supor que a falta de domínio das regras de correspondência fonema-grafema de um modo geral pode causar uma lentidão no tempo de processamento das crianças (e mesmo na precisão, como o que ocorreu aqui).

De maneira análoga ao que foi encontrado no trabalho de Pinheiro et al. (2008), o efeito de regularidade na precisão na leitura ocorreu tanto quando se considerou a direção da classificação de regularidade das palavras para a leitura quanto para a escrita. Esse achado facilita o estudo do efeito de regularidade no português, uma vez que é possível encontrar esse efeito simultaneamente nos erros e no TR controlando-se a classificação para a escrita das palavras. $\mathrm{O}$ efeito na precisão é mais fraco com 
a adoção da classificação para a escrita em relação à leitura, mas ainda mostra-se presente e, para a pesquisa, esta é uma boa notícia, uma vez que permite reduzir o número de variáveis a serem controladas pelo pesquisador.

Os efeitos de série e de extensão apareceram na direção esperada em ambos os modelos de precisão e de TR. É interessante notar que, em relação ao efeito de extensão, o aumento do TR para cada acréscimo de letra na palavra encontrado neste trabalho $(63 \mathrm{~ms})$ se assemelhou ao que foi encontrado por Pinheiro (1999). A autora descobriu que, para cada acréscimo de letra na palavra, houve um aumento de 1 a $70 \mathrm{~ms}$ no TR.

$\mathrm{O}$ fato de a criança fazer pré-escola foi um fator relevante para a precisão. As crianças com pré-escola têm, em média, um ano a mais de escolarização, e isso parece ter um efeito benéfico para o desempenho na tarefa de leitura. Além disso, e em consonância com os achados de Zevin e Seidenberg (2002), a idade de aquisição da leitura foi um fator de grande importância para o desempenho. Apesar de ter sido utilizada uma avaliação subjetiva e retrospectiva desse fator (a saber, a heteroavaliação dos pais), estes resultados apontam para a necessidade de mais estudos que considerem este fato (de preferência estudos longitudinais).

Contrariando as expectativas, não houve efeito de gênero na leitura. $\mathrm{O}$ efeito de gênero tem sido constantemente relatado em tarefas que medem habilidades verbais, sendo que as mulheres geralmente apresentam melhores desempenhos do que os homens (Chiu \& Mcbride-Chang, 2006). No entanto, os resultados aqui apresentados estão em consonância com o ponto de vista de outros autores (p. ex., White, 2007) de que as diferenças de gênero têm sido superestimadas, ou seja, de que as diferenças de gênero, quando aparecem, podem não ter uma significância prática, uma vez que os tamanhos dos efeitos são geralmente pequenos.

Nenhuma das medidas de inteligência utilizadas foi importante para o desempenho na leitura, o que está de acordo com o esperado (p. ex., Toth \& Siegel, 1994). A exceção constituiu a compreensão verbal (medida em QI), que se mostrou um fator relevante tanto para a precisão quanto para o TR. Esses resultados estão em desacordo com o que foi obtido por Dal Vesco et al. (1998) e Maia e Fonseca (2002) com crianças brasileiras. No entanto, uma diferença fundamental entre estes estudos e o presente trabalho é o fato de termos utilizado aqui o WISC-III, que apresenta adaptação e normas para nossa população, enquanto os demais utilizaram o WISC. Existe a possibilidade de que a compreensão verbal, medida pelo WISC-III, tenha captado o conhecimento verbal geral das crianças as quais são importantes para o desempenho na leitura. Por exemplo, em estudo conduzido com 73 crianças com risco genético para dislexia, foi demonstrado que estas não se diferiram do grupo de controle em termos de inteligência não-verbal - assim como no pre- sente estudo - mas tiveram um desempenho mais fraco em tarefas de fala e de processamento da linguagem, particularmente a compreensão do vocabulário e a nomeação (Gallagher, Frith, \& Snowling, 2000). De fato, vários estudos têm apontado que crianças com dificuldade na aprendizagem da leitura e da escrita apresentam problemas com tarefas que medem habilidades lingüístico-verbais e fonológicas (ver Snowling, 2004, para uma revisão).

O único fator social que interferiu na leitura da amostra foi a escolaridade da mãe, que mostrou uma relação negativa com a precisão (ou seja, os filhos de mulheres altamente escolarizadas apresentaram pior precisão na leitura do que daquelas que apresentam baixo nível de escolaridade). Não foram encontrados estudos que reportassem dados que se assemelhassem aos resultados aqui encontrados. De qualquer forma, é possível que o efeito se limite à amostra do presente estudo a qual, a pesar de heterogênea e de grande tamanho, permanece sendo uma amostra de conveniência. Sendo assim, a interpretação desta relação inusitada merece um estudo à parte, que avalie de maneira mais ampla o contexto social da criança e sua relação com o desempenho acadêmico.

Um fato interessante foi que o nível socioeconômico da família da criança não interferiu na proficiência da amostra. Isto é um resultado bastante positivo para a educação, uma vez que estamos acostumados a ver na literatura dados que apontam para diferenças socioeconômicas importantes em várias áreas de domínio cognitivo (p. ex., Duncan \& Seymour, 2000; Soares, 2004). No entanto, autores relatam diferenças socioeconômicas grupais, enquanto que no presente estudo consideramos diferenças individuais (representadas pela renda da família e não, por exemplo, pelo tipo de escola). Existe a possibilidade de que os fatores grupais ainda sejam importantes para a manifestação de diferenças em habilidades cognitivas, devido ao efeito do grupo de pares (Hanuschek et al., 2003, citados por Soares \& Collares, 2006). Uma vez que um maior nível socioeconômico propicia o acesso a vários tipos de recursos culturais pela criança, e que as escolas particulares concentram estudantes com este perfil, poderia haver um efeito do nível socioeconômico na proficiência do grupo de alunos, em contraposição a um efeito anulado ou inexistente do nível socioeconômico quando se considera o indivíduo de maneira isolada (Soares \& Collares, 2006).

Em função do que foi exposto, fica evidente a importância deste estudo para a avaliação da leitura em voz alta no português. Além de ter mostrado que todos os fatores tradicionalmente considerados relevantes para a proficiência na leitura contribuíram, de fato, para os modelos criados, este trabalho apontou novas fontes de influência (como a compreensão verbal e a escolaridade da mãe). Além disso, contribuímos com questões teóricas importantes para a avaliação cognitiva da leitura ao demonstrar que o número de categorias pode afetar a 
emergência e a extensão do efeito de regularidade e que o controle da regularidade do ponto de vista da escrita pode ser suficiente para a obtenção deste efeito. Desta forma, os resultados aqui apresentados certamente indicarão um caminho para futuras pesquisas que busquem criar instrumentos diagnósticos para as dificuldades específicas de aprendizagem que acometem as crianças em fase de desenvolvimento da leitura.

\section{Referências}

Associação Brasileira de Empresas de Pesquisa. (2003). Critério de classificação econômica Brasil. Retrieved April 25, 2009, from http://www.abep.org/codigosguias/ ABEP_CCEB_2003.pdf

Bayliss, D. M., Jarrold, C., Baddeley, A. D., \& Leigh, E. (2005). Differential constraints on the working memory and reading abilities of individuals with learning difficulties and typically developing children. Journal of Experimental Child Psychology, 92, 76-99.

Badian, N. A. (1994). Do dyslexic and other poor readers differ in reading-related cognitive skills? Reading and Writing, 6(1), 45-63.

Carvalho, M. P. (2004). Quem são os meninos que fracassam na escola? Cadernos de Pesquisa, 34(121), 11-40.

Castles, A., \& Coltheart, M. (2004). Is there a causal link from phonological awareness to success in learning to read? Cognition, 91, 77-111.

Chiu, M. M., \& Mcbride-Chang, C. (2006). Gender, context, and reading: A comparison of students in 43 countries. Scientific Studies of Reading, 10(4), 331-362.

Coltheart, M., \& Rastle, K. (1994). Serial processing in reading aloud: Evidence for dual models of reading. Journal of Experimental Psychology: Human Perception and Performance, 20(6), 1197-1211.

Coltheart, M., Rastle, K., Perry, C., Langdon, R., \& Ziegler, J. (2001). DRC: A dual route cascaded model of visual word recognition and reading aloud. Psychological Review, 108(1), 204-256.

Dal Vesco, A., Mattos, D., Benincá, C. R. S., \& Tarasconi, C. (1998). Correlação entre WISC e rendimento escolar na escola pública e na escola particular. Psicologia: Reflexão $e$ Crítica, 11(3), 481-495.

de Jong, P. F. (1998). Working memory deficits of reading disabled children. Journal of Experimental Child Psychology, 70, 75-96.

Duncan, L. G., \& Seymour, P. H. K. (2000). Socio-economic differences in foundation-level literacy. British Journal of Psychology, 91, 145-166.

Fuchs, D., \& Yung, C. (2006). On the irrelevance of intelligence in predicting responsiveness to reading instruction. Exceptional Children, 73(1), 8-30.

Gallagher, A., Frith, U., \& Snowling, M. (2000). Precursors of literacy delay among children with genetic risk of dyslexia. Journal of Child Psychology and Psychiatry, 41(2), 203213.

Gerber, A. (1996). Problemas de aprendizagem relacionados à linguagem: Sua natureza e tratamento (S. Costa, Trad.). Porto Alegre, RS: Artes Médicas.
Godoy, D. (2001). Testes de consciência fonológica e suas relações com a aprendizagem da leitura no português. Dissertação de Mestrado não-publicada, Universidade Federal de Santa Catarina, Florianópolis, SC.

Godoy, D. M. A. (2005). Aprendizagem inicial da leitura e da escrita no português do Brasil: Influência da consciência fonológica e do método de alfabetização. Tese de Doutorado não-publicada, Universidade Federal de Santa Catarina, Florianópolis, SC.

Hu, F. B., Goldberg, J., Hedeker, D., Flay, B. R., \& Pentz, M. A. (1998). Comparison of population-averaged and subjectspecific approaches for analyzing repeated binary outcomes. American Journal of Epidemiology, 147(7), 694-703.

Johnson, W. Bouchard, T. J., Jr., Segal, N. L., \& Samuels, J. (2005). General intelligence and reading performance in adults: Is the genetic factor structure the same as for children? Personality and Individual Differences, 38, 1413-1428.

Leather, C. V., \& Henry, L. A. (1994). Working memory span and phonological awareness tasks as predictors of early reading ability. Journal of Experimental Child Psychology, $58,88-111$.

Lúcio, P. S. (2008). Investigação psicométrica de uma tarefa de leitura em voz alta de palavras isoladas. Dissertação de Mestrado não-publicada, Universidade Federal de Minas Gerais, Belo Horizonte, MG.

Lúcio, P. S., \& Pinheiro, A. M. V. (in press). Vinte anos de estudo da avaliação cognitiva de leitura no português: uma revisão de literatura. Psicologia: Reflexão e Crítica, 24(1).

Maia, A. C. B., \& Fonseca, M. L. (2002). Quociente de inteligência e aquisição de leitura: Um estudo correlacional. Psicologia: Reflexão e Crítica, 15(2), 261-270.

McCann, R., \& Besner, D. (1987). Reading pseudohomophones: Implications for models of pronunciation assembly and the locus of word-frequency effects in naming. Journal of Experimental Psychology: Human Perception and Performance, 13(1), 14-24.

Morais, J. (1996). A arte de ler. São Paulo, SP: Editora da Universidade Estadual Paulista.

Neisser, N., Boodoo, G., Bouchard, T. J., Jr., Boykin, A. W., Brody, N., Ceci, S. J., et al. (1996). Intelligence: Knowns and unknowns. American Psychologist, 51(2), 77-101.

Parente, M. A. M. P., Silveira, A., \& Lecours, A. R. (1997). As palavras do português escrito. In A. R. Lecours \& M. A. M. Parente (Eds.), Dislexia: Implicações do sistema de escrita do português (pp. 41-55). Porto Alegre, RS: Artes Médicas.

Pinheiro, A. M. V. (1999). Cognitive assessment of competent and impaired reading in Scottish and Brazilian children. Reading and Writing: An Interdisciplinary Journal, 11, 175211.

Pinheiro, A. M. V. (2006). Leitura e escrita: Uma abordagem cognitiva (2. ed.). Campinas, SP: Livro Pleno.

Pinheiro, A. M. V. (2007a). Avaliação de competências psicológicas da população infanto-juvenil de Belo Horizonte: Inteligência e habilidades de leitura e escrita (FAPEMIG DC/SOT No. 1806/2007). Belo Horizonte, MG: Universidade Federal de Minas Gerais.

Pinheiro, A. M. V. (2007b). Banco de palavras de baixa freqüência de ocorrência, para crianças brasileiras da $1^{\text {a }}$ à $4^{\mathrm{a}}$ série do Ensino Fundamental, classificadas em termos de estrutura silábica, número de letras e regularidade para leitura e para escrita. In I. Sim-Sim \& F. L. Vianna, Para ava liação do desempenho da leitura. Portugal, Lisboa: Ministério da Educação. 
Pinheiro, A. M. V., Lúcio, P. S, \& Silva, D. M. R. (2008). O efeito de regularidade grafema-fonema e fonema-grafema na leitura em voz alta de palavras isoladas. Psicologia: Teoria e Prática, 10(2), 16-20.

Pinheiro, A. M. V., \& Rothe-Neves, R. (2001). Avaliação cognitiva da leitura: As tarefas de leitura em voz alta e ditado. Psicologia: Reflexão e Crítica, 14(2), 399-408.

Raven, J., Raven, J. C., \& Court, J. H. (1993). Manual das Matrizes Progressivas de Raven: Escala especial (L. A. Angelini, I. C. B. Alves, E. M. Custódio, W. F. Duarte, \& J. L. M. Duarte, Trads. e adaptação). São Paulo, SP: Centro Editor de Testes e Pesquisas em Psicologia.

Salles, J. F. (2005). Habilidades e dificuldades de leitura e escrita em crianças de $2^{a}$ série: Abordagem neuropsicológica cognitiva. Tese de Doutorado não-publicada, Universidade Federal do Rio Grande do Sul, Porto Alegre, RS.

Salles, J. F., \& Parente, M. A. M. P. (2002). Processos cognitivos na leitura de palavras em crianças: Relações com compreensão e tempo de leitura. Psicologia: Reflexão $e$ Crítica, 15(2), 321-331.

Salles, J. F., \& Parente, M. A. M. P. (2007). Avaliação da leitura e da escrita de palavras em crianças de $2^{\mathrm{a}}$ série: Abordagem neuropsicológica cognitiva. Psicologia: Reflexão $e$ Crítica, 20(2), 220-228

Snowling, M. (2004). Dislexia desenvolvimental: Uma introdução e visão teórica geral (M. F. Lopes, Trad.). In M. Snowling \& J. Stackhouse (Eds.), Dislexia, fala e linguagem: Um manual profissional (pp. 11-21). Porto Alegre, RS: Artmed.

Siegel, L. S., \& Linder, B. (1984). Short-term memory process in children with reading and arithmetic disabilities. Developmental Psychology, 20, 200-207.

Soares, J. F. (2004). O efeito da escola no desempenho cognitivo de seus alunos. REICE - Revista Eletrónica Iberoamericana sobre Calidad, Eficacia y Cambio en Educación, 2(2), 83104.

Soares, J. F., \& Collares, A. C. M. (2006). Recursos familiares e o desempenho cognitivo dos alunos do ensino básico brasileiro. DADOS: Revista de Ciências Sociais, 49(3), 615650.

Stanovich, K. E., Cunningham, A., \& Freeman, D. (1984). The relationship between early reading acquisition and word decoding with and without context: A longitudinal study of first-grade children. Journal of Educational Psychology, 76, 668-677.

Steensel, R. V. (2006). Relations between socio-cultural factor, the home literacy environment and children's literacy development in the first years of primary education. Journal of Research in Reading, 29(4), 367-382.

Stein, L. M. (1994). TDE - Teste de Desempenho Escolar. São Paulo, SP: Casa do Psicólogo.

Sucena, A., \& Castro, S. L. (2005) Estratégias fonológicas e ortográficas na aprendizagem da leitura do português europeu. Anales de la Revista de Psicologia General y Aplicada, 10(3). Retrieved March 10, 2009, from http:// www.fedap.es/IberPsicologia/iberpsi10/indiceip10-3.htm

Toth, G., \& Siegel, L. S. (1994). A critical evaluation of the IQ-Achievement discrepancy based definition of dyslexia. In K. P. van den Boss, L. S. Siegel, D. J. Bakker, \& D. L. Share (Eds.), Current directions in dyslexia research (pp. 45-70). Lisse, Netherlands: Swets \& Zeitlinger.
Wechsler, D. (1991). WISC-III: Escala de Inteligência Wechsler para Crianças: Manual (3. ed., V. L. M. de Figueiredo, Adaptação e padronização). São Paulo, SP: Casa do Psicólogo.

White, B. (2007). Are girls better readers than boys? Which boys? Which girls? Canadian Journal of Education, 30(2), 554-581.

Zevin, J. D., \& Seidenberg, M. S. (2002). Age of acquisition effects in word reading and other tasks. Journal of Memory and Languagem, 47, 1-29.
Recebido: $27 / 04 / 2009$

$1^{a}$ revisão: $29 / 06 / 2009$ Aceite final: $12 / 07 / 2009$ 